\title{
Propiedades reológicas y de adsorción de agua de harina extrudida de arroz y bagazo de cebada
}

\author{
Fernanda Ferreira Cardoso ${ }^{1}$, Diego Palmiro Ramirez Ascheri ${ }^{2}$, Carlos Wanderlei Piler de Carvalho ${ }^{3}$
}

\section{RESUMEN}

El bagazo de cebada procedente de la industria cervecera se utiliza comúnmente en la alimentación animal, pero por medio de un proceso adecuado puede servir como alternativa en las formulaciones de productos alimentares ricos en fibra y proteína. La combinación de bagazo de cebada y arroz (82:18) fue procesada por extrusión para obtener la harina mixta extrudida de bagazo y arroz, de la cual se determinaron las propiedades reológicas y de adsorción de agua, cuyos valores fueron comparados con la harina de arroz cruda. Los modelos matemáticos de Oswin, Smith, Peleg (1988) y Peleg (1993) fueron utilizados para explicar las propiedades de absorción en diferentes temperatura $\left(25,30,35\right.$ y $\left.45^{\circ} \mathrm{C}\right)$ y actividades de agua (0.11 a 0.97). Peleg (1993) fue el modelo que mejor describe las curvas de absorción de agua. El perfil de viscosidad aparente de la harina extrudida es similar al de harina de arroz crudo; no presenta viscosidad inicial pero se caracteriza por tener menor temperatura de pasta, quiebra de viscosidad y tendencia a la retrogradación.

Palabras clave: residuos, cebada, isoterma, extrusión termoplástica.

\section{RESUMO}

\section{Propriedades reológicas e de adsorção de água de farinha extrudada de arroz e bagaço de cevada}

O bagaço de cevada cervejeira é comumente utilizado na alimentação animal, porém, por meio de um processamento adequado pode servir em formulação alternativa de produtos alimentares ricos em fibras e proteínas. A combinação de bagaço de cevada e arroz (82:18) foi processada por extrusão para obter-se a farinha mista extrudada de bagaço e arroz, da qual se determinaram as propriedades reológicas e de absorção de água, cujos valores foram comparados com os da farinha de arroz crua. Os modelos matemáticos de Oswin, Smith, Peleg (1988) e Peleg (1993) foram utilizados para explicar as propriedades de absorção em diferentes temperaturas $\left(25,30,35\right.$ e $\left.45^{\circ} \mathrm{C}\right)$ e atividades de água $(0,11-0,97)$. Peleg (1993) foi o modelo que melhor descreveu as curvas de absorção de água. O perfil de viscosidade aparente da farinha extrudada é semelhante ao da farinha de arroz crua; não apresenta viscosidade inicial, caracteriza-se, porém, por ter menor temperatura de pasta, quebra de viscosidade e tendência à retrogradação.

Palavras-chave: resíduos, cevada, isoterma, extrusão termoplástica.

\footnotetext{
Enviado el 06/11/2012 y aprobado el 21/05/2014.

${ }^{1}$ Ingeniera de Alimentos, Master. Carretera, Br 153, 3.105b, Fazenda Barreiro do Meio, 75132-903, Caixa Postal 459, Anápolis, Goiás, Brasil. fe.ferreira07@gmail.com ${ }^{2}$ Ingeniero de Alimentos, Doctor. Carretera Br 153, 3.105b, Fazenda Barreiro do Meio, 75132-903, Caixa Postal 459, Anápolis, Goiás, Brasil. ascheridpr@gmail.com (autor de correspondencia).

${ }^{3}$ Ingeniero Agronomo, Doctor. Embrapa Agroindústria de Alimentos, Avenida de las Américas, 29501, 23020-470, Guaratiba, Rio de Janeiro, Brasil. cwpiler@ctaa.embrapa.br
} 


\section{INTRODUCCIÓN}

El bagazo de cebada es un desecho industrial de la fermentación de los granos utilizados en el proceso de fabricación de la cerveza. Este representa $85 \%$ del total de los residuos generados en la industria cervecera, siendo considerado de esa manera, el más importante desecho proveniente de ese proceso. Es aprovechado como ración para animales. Entretanto, Ascheri et al. (2005) refieren que el bagazo de cebada se constituye en fibras $(20 \%)$ e proteínas (20-25\%) que, misturados con otros desechos industriales como el afrecho de arroz, por medio de un proceso adecuado, también se lo puede aprovechar en la dieta alimentar. Ascheri et al. (2006b) observaron que las propiedades físico-químicas de harinas mixtas pregelatinizadas compuestas de bagazo de jabuticaba y de arroz se diferenciaron significativamente de las respectivas harinas crudas.

La extrusión es un proceso continuo, versátil y muy utilizado en la transformación de alimentos. En los alimentos extruidos ocurren modificaciones químicas y cambios estructurales como la gelatinización del almidón (Van den Einde et al., 2005), la desnaturalización de las proteínas (Guy, 2001), la formación de complejos entre estos constituyentes y otras reacciones. Forma productos de diferentes calidades. Yu \& Wang (2007), Zanão et al. (2009) y Ascheri et al. (2010) observaron que las propiedades de pasta del arroz fueron significativamente alteradas cuando sometidas a tratamientos drásticos de calor, ocurriendo la reducción de la temperatura de pasta, disminución del tiempo para la ocurrencia del pico de viscosidad, reducción en los valores de viscosidad máxima y viscosidad final y la tendencia de retrogradación.

Así como los parámetros del proceso de extrusión (temperatura, velocidad del tornillo sin fin, humedad, etc), el contenido de fibras de las muestras también es un factor muy importante en la calidad del producto extrudido. Berglund et al. (1994) en mezclas de harinas de cebada y arroz o trigo observaron que extrudidos producidos con $100 \%$ de harina de cebada tienen su expansión limitada y alta densidad debido a su elevado contenido de fibra alimentar. La densidad del extrudido disminuye el 50\% cuando son producidos con mezcla de 50:50 de harinas de cebada y arroz. Gupta et al. (2008) también trabajaron con extrudidos a base de harina de cebada y arroz indiano y concluyeron que la disminución de la proporción cebada en formulaciones menos húmedas $(<20 \%)$ se obtienen extrudidos con mayor valor de dureza, pero, cuando se aumenta esa proporción (>30\% de cebada), manteniendo la humedad menor de $20 \%$, el producto extrudido presenta mayor índice de absorción de agua. Además, la adición de harina de cebada a las formulaciones dificulta la gelatinización del almidón, resultando productos extrudidos con baja viscosidad de empastamiento.
Almidones que sufrieron tratamiento térmico presenta mayor cantidad de sitios de adsorción (oxidrilos, $-\mathrm{OH}$ ) disponibles para formar puentes de hidrógeno con el agua (Lima et al., 2012); son mas solubles en agua fría. Si la disponibilidad de esos sitios es grande, el producto amiláceo se vuelve higroscópico en determinadas condiciones de temperatura e actividad de agua. Siendo así, el estudio de las propiedades de adsorción de agua es importante a fin de determinar el tipo de embalaje y las condiciones de almacenamiento para aumentar el tiempo de vida útil de ese producto.

Las propiedades de adsorción de agua pueden ser estudiados por medio de las isotermas que son representaciones gráficas del contenido del agua presente en el alimento analizado frente a la humedad relativa, donde el alimento se encuentra en equilibrio higroscópico con el ambiente en que se encuentra sin cambios de masa del alimento (García \& Martínez, 2006).

Las propiedades de adsorción de agua relacionan la humedad y la energía del producto en determinadas condiciones de humedad relativa y temperatura; además infieren la cantidad de energía a usar para conservar el producto en un ambiente adecuado por períodos de tiempo prolongados.

Teniendo en cuenta que las harinas y otro materiales amiláceos se modifican después del proceso de extrusión y que la adición de fibras en las formulaciones disminuye la calidad de los extrudidos en comparación con los producidos con cereales de maíz y arroz, los estudios de las propiedades reológicas y de adsorción de agua son de gran importancia por fornecer informaciones sobre el comportamiento de las pastas de las harinas producidas de los extrudidos, en función de la temperatura y cizallamiento, y la estabilidad de las harinas, en función del tiempo, temperatura y humedad.

Por eso, este trabajo tuvo por objetivo evaluar las propiedades reológicas y de adsorción de agua de harina mixta de arroz y bagazo de cebada pre-gelatinizada por extrusión a partir de la adsorción de agua, en función del tiempo, de la temperatura y de la actividad del agua.

\section{MATERIAL E MÉTODOS}

El experimento se realizó en el Laboratorio de Propiedades Físicas de Productos Vegetales de Ciencias Exactas y Tecnológicas de Goiás (Anápolis-GO, Brasil) y en el Laboratorio de extrusión da Embrapa Agroindústria de Alimentos (Rio de Janeiro-RJ, Brasil).

El bagazo de cebada con humedad inicial de $298 \%$ en base seca fue donado por la AmBev (Anápolis-GO). Muestras de bagazo fueron deshidratados en estufa con recirculación de aire (Marconi, Marconi, modelo MA03/5, Piracicaba, Brasil), a $60^{\circ} \mathrm{C}$ por 7 días (d) hasta alcanzar una humedad de aproximadamente $7 \%$ en base seca; molido en 
molino de cuchillos (Marconi, MA580, Piracicaba, Brasil) y tamizado hasta partículas menores de $300 \mu \mathrm{m}$, obteniéndose la harina de bagazo de cebada (HBC).

El arroz fue adquirido en el comercio local con humedad inicial de $12 \%$ en base seca. Muestras de ese arroz también fueron molidos y tamizados hasta partículas finas, obteniéndose la harina de arroz (HA).

En bolsas de polietileno de alta densidad, las harinas anteriormente obtenidas fueron mezcladas en proporciones de HA y HBC de 82:18 e añadidas de $19 \%$ de agua (en base a la cantidad de la mezcla), medidas que fueron seleccionados en estudios preliminares. Las bolsas fueron amarradas y las mezclas fueron homogenizadas manualmente y acondicionadas a $10{ }^{\circ} \mathrm{C}$ por $24 \mathrm{~h}$ para una distribución y absorción más uniforme del agua.

Teniendo en cuenta la dificultad de extrudir fibras (produce mayor atrito en el interior del cañón de extrusión), los parámetros de extrusión fueron escogidos en estudio previo a este trabajo. Se ha utilizado un extrusor Brabender (Duisburg, Alemanha), modelo 19/20 DN, compuesto de un único tornillo sin fin con tasa de compresión de 1:3, cuya rotación fue de $180 \mathrm{rpm}$. El diámetro de la matriz fue de $0,3 \mathrm{~cm}$ y la tasa de alimentación de $5 \mathrm{~kg} \mathrm{~h}^{-1}$. Las temperaturas de calentamiento de las zonas 1 a 3 fueron fijadas en 80,100 y $152^{\circ} \mathrm{C}$, respectivamente. El material extrudido fue colectado después que el proceso alcanzó el equilibrio visual obtenido en la lectura del torque de la extrusora (aproximadamente $15 \mathrm{~N} \mathrm{~m}$ ). La colecta fue manual, cortando el material extruido en tamaño aproximado de $30 \mathrm{~cm}$ de largura, acondicionado en bandejas de aluminio y deshidratado en estufa con recirculación de aire a $60{ }^{\circ} \mathrm{C}$ hasta alcanzar 3 a 7\% de humedad en base seca. A seguir, el extrudido fue molido en molinos de disco Perten 3300 (Perten Instruments, Huddinge, Finlãndia) y de rolos Brabender Junior (Brabender, Duisburg, Alemanha), obteniéndose, así, las harinas mixtas extrudidas (FME). Después del molido, las FME fueron acondicionadas en sacos plásticos, debidamente etiquetadas y almacenadas a la temperatura ambiente.

El análisis de la viscosidad de la HA y HME fue realizada según la metodología del manual Rapid Visco Analyzer (RVA 4, Newport Scientific PTY LTD, Sydney, Australia). La lectura fue realizada en suspensiones acuosas de $12 \%(\mathrm{~m} / \mathrm{v})$ de las harinas de arroz y extrudida, previamente corregidas para 14\% de humedad (en base húmeda). El RVA fue programado como sigue: por 2 min a la temperatura de $25^{\circ} \mathrm{C}$, calentamiento a una tasa de 14 $\min ^{-1}$ hasta $95^{\circ} \mathrm{C}$, manutención de la pasta por $3 \mathrm{~min}$ y enfriamiento a la misma tasa hasta alcanzar $25^{\circ} \mathrm{C}$. Se evaluó el perfil de cocción de las pastas por la observación de la temperatura de formación de pasta $\left(\mathrm{T}_{\mathrm{e}}\right)$, viscosidad máxima (Vmax), viscosidad mínima (Vmin) durante el enfriamiento, viscosidad de la quiebra ( $\mathrm{BD}=\mathrm{Vmax}-\mathrm{Vmin})$, viscosidad final (Vfin) de las pastas y tendencia a la retrogradación ( $\mathrm{SB}=\mathrm{V}$ fin-Vmin). Los resultados de viscosidad aparente fueron registrados en centipoise $(\mathrm{cP})$.

El proceso de adsorción se dio usando el método gravimétrico estático de acuerdo con el Proyecto COST 90 modificado por Ascheri et al. (2003). Las soluciones saturadas de sales fueron colocados en frascos de vidrio que cuando tapados herméticamente generaron ambientes con diferentes valores de actividad de agua $\left(\mathrm{A}_{\mathrm{w}}\right)$ (Tabla 1), variando de 0.112 a 0.973 , en temperaturas de 25 a $45^{\circ} \mathrm{C}$.

La masa inicial de las muestras fue aproximadamente de $0,5 \mathrm{~g}$ con $1 \%$ de humedad en base seca. Durante el proceso de adsorción, la masa de las muestras se detectó en balanza semi analítica (Gehaka, BG 400, Brasil) a cada 3 h o más, hasta no haber variación de masa, es decir, cuando tres o más medias sean semejantes. La humedad de las muestras $\left(\mathrm{X}_{\mathrm{e}, \mathrm{t}}\right.$, en $\%$ base seca) para un determinado tiempo de adsorción, se calculó con:

$X_{e, t}=100\left(\frac{m_{e}-m_{i}}{m_{i}}\right)_{t}$

en que: $m_{i}$ - masa inicial de la muestra seca $(\mathrm{kg}), \mathrm{m}_{\mathrm{e}}$ - masa de la muestra húmeda para un determinado tiempo de adsorción (kg) ), y t - tiempo de adsorción

Los modelos matemáticos de Oswin (Chirife \& Iglesias, 1978), Smith (Labuza et al., 1985) y Peleg (1993) de la Tabla 2 se aplicaron para estimar la humedad de equilibrio en función de la actividad del agua. Los datos de $\mathrm{X}_{\mathrm{e}, \mathrm{t}}$ utilizados para este fin fueron aquellos calculados por la media aritmética de las tres o más medias de $\mathrm{X}_{\mathrm{e}, \mathrm{t}}$ semejantes observadas en el patamar de las curvas de la Figura 1. Las medias $\left(\mathrm{X}_{\mathrm{e}, \mathrm{t}}\right)$ se muestran en la Tabla 1.

El modelo de Peleg (1988) (Tabla 2) fue transformado a su forma lineal (Ecuación 6) para estimar los valores de humedad de equilibrio directamente de los datos experimentales, en función del tiempo de adsorción.

$\frac{t}{X_{e, t}-X_{0}}=r_{1}+r_{2} \mathrm{t}$

La humedad de equilibrio estimado por el modelo de Peleg (1988) $\left(\mathrm{X}_{\mathrm{e}, \mathrm{P}}\right)$ puede ser obtenido por la propia Ecuación (6) cuando el tiempo tiende al infinito, resultando en:

$X_{e, P}=X_{0}+\frac{1}{r_{2}}$

Inicialmente, el valor de $\mathrm{X}_{0}$ es igual a cero, por lo tanto, $\mathrm{X}_{\mathrm{e}, \mathrm{P}}=\frac{1}{\mathrm{r}_{2}}$.

El diseño experimental fue completamente al azar con tres repeticiones. Las variables independientes fueron actividad de agua y tiempo de adsorción y las variables 
respuesta fueron las humedades de equilibrio $\mathrm{X}_{\mathrm{e}, \mathrm{aw}} \mathrm{y} \mathrm{X}_{\mathrm{e}, \mathrm{t}}$, respectivamente. Estadísticamente fue analizado el ajuste de los modelos matemáticos de Oswin, Smith e Pele (1993) (Ecuaciones 2 a 4, respectivamente) a los datos experimentales de humedad de equilibrio, en función de la actividad de agua, en las diferentes temperaturas utilizadas; aplicándose el análisis de variancia para regresión no linear al nivel de 5\% de probabilidad (Elian, 1988), y los ensayos estadísticos error relativo medio (ERM) y error estándar del estimado (SE), al mismo nivel de probabilidad anterior.

La confirmación de la linealidad del modelo de Peleg (1988) linealizado se efectuó aplicando análisis de variancia para regresión linear al nivel de 5\% de probabilidad, solucionado por el método de los mínimos cuadrados (Montgomery \& Runger, 2003). Las constan- tes del modelo fueron evaluados por las estadísticas coeficiente de determinación $\left(\mathrm{R}^{2}\right)$, y $\mathrm{SE}$.

Los análisis de regresión y las estadísticas se efectuaron con el programa Statistica 8.0, a 95\% de confianza.

\section{RESULTADOS E DISCUSSÃO}

De acuerdo con Shuey \& Tipples (1980) los gránulos de almidón cuando sometidos a un severo tratamiento térmico los puentes de hidrógeno que unen las moléculas en red micelar se quiebran, favoreciendo a la capacidad de hidratación de las cadenas glucosídicas, así, se tiene un proceso de hinchamiento irreversible, que irá a reflejar al grado de gelatinización del almidón cuando a temperaturas frías, y sus características de adsorción de agua

Tabla 1. Actividades de agua $\left(\mathrm{A}_{\mathrm{w}}\right)$, temperaturas de adsorción $(\mathrm{T})$ y respectivos sales saturados $(\mathrm{Sal})$ utilizados para la determinación de la humedad de equilibrio $\left(\overline{\mathrm{X}}_{\mathrm{e}, \mathrm{t}}\right)$ de la harina mixta extrudida de bagazo de cebada y arroz. Coeficientes $\mathrm{r}_{1}$ y $\mathrm{r}_{2}$ y humedad de equilibrio estimado $\left(\mathrm{X}_{\mathrm{e}, \mathrm{P}}\right)$ por el modelo de Peleg (1988) linealizado.

\begin{tabular}{|c|c|c|c|c|c|c|c|c|c|}
\hline \multirow{2}{*}{$\mathbf{T}\left({ }^{\circ} \mathbf{C}\right)$} & \multirow{2}{*}{ Sal } & \multirow{2}{*}{$\mathbf{A}_{\mathrm{w}}$} & \multirow{2}{*}{$\bar{X}_{e, t}(\%)$} & \multicolumn{2}{|c|}{ Coeficientes } & \multirow{2}{*}{$\begin{array}{l}X_{e, P} \\
(\%)\end{array}$} & \multirow{2}{*}{$\mathbf{R}^{2}$} & \multirow{2}{*}{$\mathbf{P}(\%)$} & \multirow{2}{*}{ SE } \\
\hline & & & & $r_{1}$ & $\mathbf{r}_{2}$ & & & & \\
\hline \multirow{8}{*}{25} & $\mathrm{LiCl}$ & 0.11 & 4.13 & 0.87 & 0.23 & 4.32 & 0.990 & 4.43 & 0.29 \\
\hline & $\mathrm{KC}_{2} \mathrm{COOH}$ & 0.23 & 5.73 & 0.45 & 0.17 & 5.91 & 0.990 & 2.81 & 0.11 \\
\hline & $\mathrm{MgCl}_{2}$ & 0.33 & 7.83 & 0.19 & 0.13 & 7.98 & 0.999 & 3.05 & 0.07 \\
\hline & $\mathrm{KI}$ & 0.69 & 13.49 & 0.10 & 0.07 & 13.73 & 0.999 & 1.98 & 0.03 \\
\hline & $\mathrm{NaCl}$ & 0.75 & 14.81 & 0.07 & 0.07 & 15.03 & 0.999 & 1.65 & 0.02 \\
\hline & $\left(\mathrm{NH}_{3}\right)_{2} \mathrm{SO}_{4}$ & 0.81 & 16.32 & 0.07 & 0.06 & 16.55 & 0.999 & 1.85 & 0.02 \\
\hline & $\mathrm{KCl}$ & 0.84 & 18.07 & 0.09 & 0.05 & 18.45 & 0.999 & 0.95 & 0.02 \\
\hline & $\mathrm{K}_{2} \mathrm{SO}_{4}$ & 0.97 & 32.36 & 0.28 & 0.03 & 35.30 & 0.997 & 6.20 & 0.05 \\
\hline \multirow{8}{*}{30} & $\mathrm{LiCl}$ & 0.11 & 2.58 & 1.35 & 0.35 & 2.89 & 0.999 & 2.10 & 0.22 \\
\hline & $\mathrm{KC}_{2} \mathrm{COOH}$ & 0.22 & 4.34 & 0.71 & 0.21 & 4.66 & 0.999 & 1.37 & 0.08 \\
\hline & $\mathrm{MgCl}_{2}$ & 0.32 & 6.22 & 0.30 & 0.15 & 6.50 & 0.999 & 1.43 & 0.04 \\
\hline & $\mathrm{KI}$ & 0.68 & 12.66 & 0.12 & 0.08 & 13.14 & 0.999 & 1.46 & 0.03 \\
\hline & $\mathrm{NaCl}$ & 0.75 & 14.00 & 0.07 & 0.07 & 14.39 & 0.999 & 1.48 & 0.02 \\
\hline & $\left(\mathrm{NH}_{3}\right)_{2} \mathrm{SO}_{4}$ & 0.81 & 15.65 & 0.10 & 0.06 & 16.20 & 0.999 & 1.74 & 0.03 \\
\hline & $\mathrm{KCl}$ & 0.84 & 17.10 & 0.11 & 0.06 & 17.89 & 0.999 & 2.56 & 0.03 \\
\hline & $\mathrm{K}_{2} \mathrm{SO}_{4}$ & 0.97 & 31.93 & 0.18 & 0.03 & 36.15 & 0.994 & 4.79 & 0.04 \\
\hline \multirow{8}{*}{35} & $\mathrm{LiCl}$ & 0.11 & 2.90 & 1.41 & 0.31 & 3.25 & 0.997 & 2.26 & 0.31 \\
\hline & $\mathrm{KC}_{2} \mathrm{COOH}$ & 0.21 & 4.82 & 0.50 & 0.19 & 5.17 & 0.998 & 1.72 & 0.16 \\
\hline & $\mathrm{MgCl}_{2}$ & 0.32 & 6.48 & 0.17 & 0.15 & 6.71 & 0.998 & 1.73 & 0.12 \\
\hline & KI & 0.67 & 11.76 & 0.06 & 0.08 & 12.08 & 0.998 & 1.62 & 0.07 \\
\hline & $\mathrm{NaCl}$ & 0.75 & 13.46 & 0.07 & 0.07 & 13.93 & 0.998 & 2.00 & 0.06 \\
\hline & $\left(\mathrm{NH}_{3}\right)_{2} \mathrm{SO}_{4}$ & 0.80 & 14.86 & 0.06 & 0.07 & 15.35 & 0.999 & 1.30 & 0.05 \\
\hline & $\mathrm{KCl}$ & 0.83 & 15.97 & 0.06 & 0.06 & 16.49 & 0.999 & 1.21 & 0.04 \\
\hline & $\mathrm{K}_{2} \mathrm{SO}_{4}$ & 0.97 & 29.80 & 0.19 & 0.03 & 33.33 & 0.995 & 4.80 & 0.04 \\
\hline \multirow{8}{*}{45} & $\mathrm{LiCl}$ & 0.11 & 3.04 & 0.68 & 0.31 & 3.28 & 0.999 & 0.97 & 0.06 \\
\hline & $\mathrm{KC}_{2} \mathrm{COOH}$ & 0.20 & 4.51 & 0.27 & 0.21 & 4.72 & 0.999 & 0.65 & 0.03 \\
\hline & $\mathrm{MgCl}_{2}$ & 0.31 & 6.44 & 0.08 & 0.15 & 6.57 & 0.999 & 0.71 & 0.01 \\
\hline & KI & 0.65 & 11.12 & 0.02 & 0.09 & 11.19 & 0.999 & 0.14 & 0.00 \\
\hline & $\mathrm{NaCl}$ & 0.75 & 13.26 & 0.02 & 0.07 & 13.42 & 0.999 & 0.46 & 0.01 \\
\hline & $\left(\mathrm{NH}_{3}\right)_{2} \mathrm{SO}_{4}$ & 0.80 & 14.75 & 0.04 & 0.07 & 15.11 & 0.999 & 0.67 & 0.01 \\
\hline & $\mathrm{KCl}$ & 0.82 & 15.36 & 0.05 & 0.06 & 15.80 & 0.999 & 0.26 & 0.00 \\
\hline & $\mathrm{K}_{2} \mathrm{SO}_{4}$ & 0.96 & 27.21 & 0.08 & 0.03 & 29.48 & 0.999 & 1.50 & 0.01 \\
\hline
\end{tabular}

\footnotetext{
"Medias obtenidas de tres repeticiones. $\mathrm{R}^{2}$ : coeficiente de determinación, $\mathrm{P}(\%)$ : error relativo medio, SE: error estándar del estimado.
} 
durante su almacenamiento. Sin embargo, esto ocurrió en menor intensidad con la harina mixta extrudida.

Los datos experimentales de humedad de equilibrio en función del tiempo de adsorción de agua registrados en cada solución salina y temperaturas están representados en forma de gráficos en la Figura 1. En las etapas iniciales de adsorción la humedad de equilibrio aumentó rápidamente, sin embargo, disminuyó al aumentar el tiempo hasta alcanzar un patamar, indicando que se llegó al equilibrio dinámico en cada condición de adsorción establecida.
Por la Figura 1 se notó que en las temperatura de 25, 30,35 y $45^{\circ} \mathrm{C}$, el tiempo necesario para alcanzar el equilibrio en las actividades de agua entre 0.112 y 0.840 fue disminuyendo, siendo estes de 24-27, 18-24, 12-21 y 9-15 min, respectivamente. Pero, este hecho no fue notado en $\mathrm{A}_{\mathrm{w}} \mathrm{e} \geq 0.96$. El tiempo de equilibrio no disminuyó como anteriormente, siendo estos de 95, 36, 48 y $18 \mathrm{~min}$, respectivamente. Baucour \& Daudin (2000) y confirmado por Kulchan et al. (2010) explicaron este hecho, relatando que en actividades de agua elevadas (>0.90), la transferencia
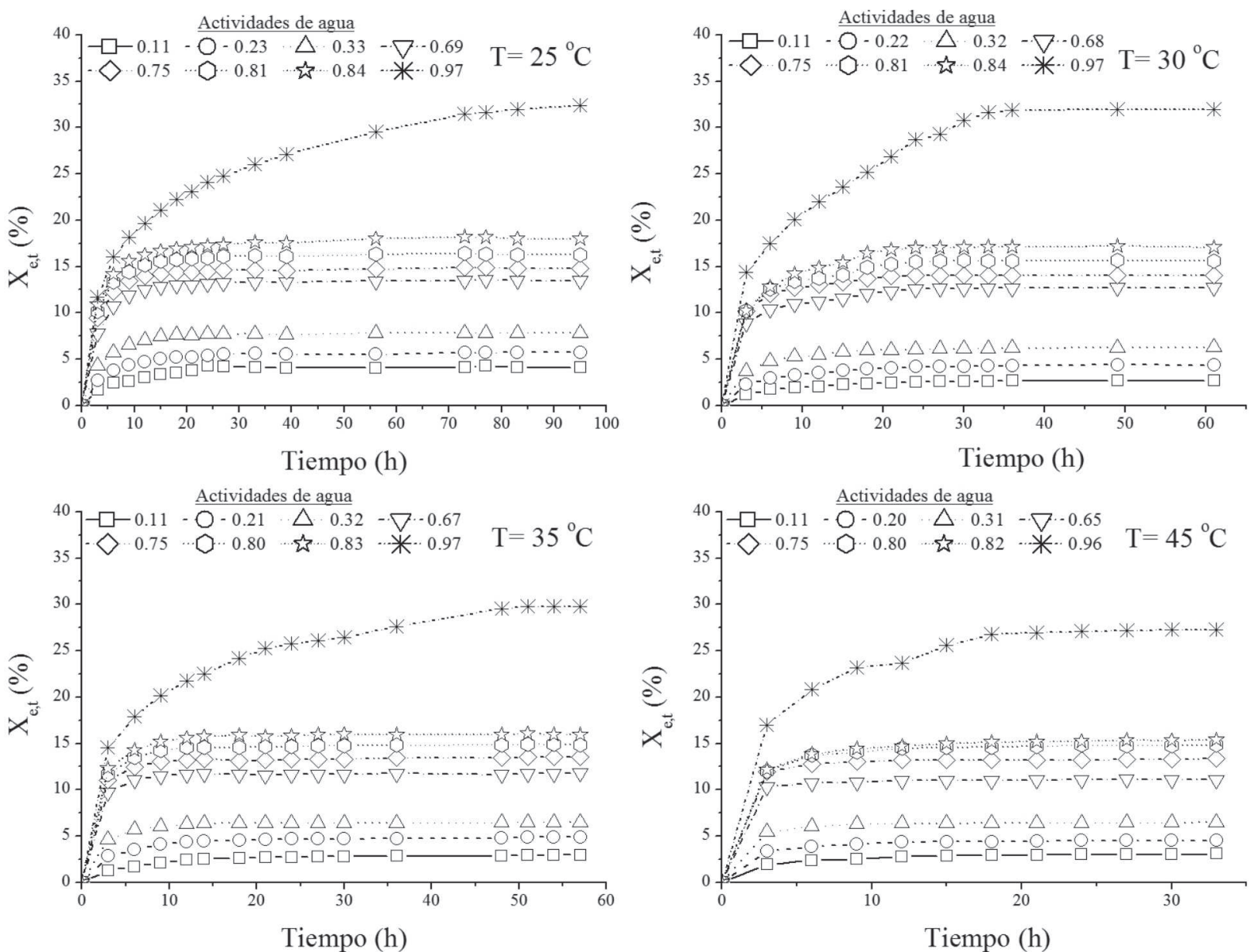

Figura 1. Variación de la humedad de equilibrio $\left(X_{e, t}\right)$ de la harina mixta extrudida de arroz y bagazo de cebada en función del tiempo de adsorción de agua, obtenidas en diferentes temperaturas $(\mathrm{T})$ y actividades de agua.

Tabla 2. Modelos matemáticos de adsorción de agua de dos y tres parámetros.

\begin{tabular}{|c|c|c|c|}
\hline Modelo & Referencia & \multicolumn{2}{|c|}{ Modelo matemático de adsorción } \\
\hline Oswin & Chirife \& Iglesias (1978) & $\bar{X}_{e, t}=a\left(\frac{A_{w}}{\left(1-A_{w}\right.}\right)^{b}$ & (2) \\
\hline Smith & Labuza et al. (1985) & $\bar{X}_{e, t}=a-b \ln \left(1-A_{w}\right)$ & (3) \\
\hline Peleg & Peleg (1993) & $\overline{\mathrm{X}}_{\mathrm{e}, \mathrm{t}}=\mathrm{k}_{1} \mathrm{~A}_{\mathrm{w}}^{\mathrm{n}_{1}}+\mathrm{k}_{2} \mathrm{~A}_{\mathrm{w}}^{\mathrm{n}_{2}}$ & (4) \\
\hline Peleg & Peleg (1988) & $X_{e, t}=X_{0} \pm \frac{t}{\left(r_{1}+r_{2} t\right)}$ & $(5)$ \\
\hline
\end{tabular}

(\% base seca ): media aritmética de la humedad de equilibrio en función del tiempo, $\mathrm{X}_{\mathrm{e}, \mathrm{t}}(\%$ base seca ): humedad de equilibrio en función del tiempo, $\mathrm{X}_{0}$ (\% base seca ): humedad de equilibrio inicial, $\mathrm{A}_{\mathrm{w}}$ (adimensional): actividad de agua, a, b, $\mathrm{k}_{1}, \mathrm{k}_{2}, \mathrm{n}_{1}$ y $\mathrm{n}_{2}$ : constantes características de las ecuaciones de adsorción de agua. $\mathrm{r}_{1}$ y $\mathrm{r}_{2}$ : coeficientes del modelo de Peleg (1988). 
de masa es muy lenta, lo que se hace difícil alcanzar el equilibrio en $\mathrm{A}_{\mathrm{w}} \mathrm{e} \geq 0.9$. En el presente estudio, probablemente la muestra utilizada $30{ }^{\circ} \mathrm{C}$ debe estar compuesta con mayor proporción de almidón proveniente de la harina de arroz siendo más higroscópico que las fibras del bagazo de cebada, por lo tanto, más rápidamente adsorbe agua y llega al equilibrio dinámico.

Las estadísticas (Tabla 1) aplicadas demostraron que el modelo de Peleg (1988) linealizado se ajustó adecuadamente a los datos experimentales obtenidos en función del tiempo de adsorción (Figura 1), con valores de $\mathrm{R}^{2} \geq$ 0.99; $\mathrm{P}(\%) \leq 6.20$ y $\mathrm{SE} \leq 0.31$. Para Cunningham et al. (2007) estas estadísticas son satisfactorias, por tanto, el modelo matemático de Peleg (1988) puede estimar los valores de humedad de equilibrio de las harinas mixtas extrudidas de bagazo de cebada y arroz, en función del tiempo de adsorción, cuyos resultados estimados están en la Tabla $1\left(\mathrm{X}_{\mathrm{e}, \mathrm{P}}\right)$.

Las constantes $r_{1}$ y $r_{2}$ también pueden ser vistos en la Tabla 1. Generalmente, sus valores tienden a disminuir con el aumento de la actividad del agua y viceversa; ellas están asociadas a la transferencia de masa (Turhan et al., 2002). Cuanto menores son los valores de $\mathrm{k}_{1} \mathrm{y} \mathrm{k}_{2}$ mayor es la tasa inicial y la capacidad de adsorción de agua de la harina, respectivamente. Con excepciones de $\mathrm{r}_{1}$ que experimenta un ligero aumento con valores de $\mathrm{A}_{\mathrm{w}} \geq 0.8$, significando que en esas $\mathrm{A}_{\mathrm{w}}$ la harina presenta una menor tasa inicial de adsorción de agua, debido a una lenta transferencia de masa a actividades de agua elevadas.

Los datos experimentales de $\mathrm{X}_{\mathrm{e}}$ en función de la actividad del agua para las temperaturas de adsorción estudiadas están en la Tabla 1 y Figura 3.

Las isotermas de la Figura 2 presentan forma sigmoidal del tipo II según la clasificación de la International Union of Pure and Applied of Chemistry (1985), típicas de superficies hidrofílicas tales como, harinas de arroz pregelatinizadas por extrusión termoplástica (Ascheri et al., 2003), microcápsulas de lactoferrina (Maia et al., 2004), harinas extrudidas de amaranto, arroz y maíz (Ascheri et al., 2005), almidón de rizomas del lírio del campo (Ascheri et al., 2009), harinas crudas y pregelatinizadas de arroz (Lima et al., 2012) y otros.

Se puede verificar por el teste F de la Tabla 3, que la regresión no lineal contribuyó significativamente para el ajuste de los modelos de Oswin, Smith y Peleg (1993) a los datos experimentales de $\mathrm{X}_{\mathrm{e}}$. La confiabilidad de la adecuación fue elevada en todas las isotermas, siendo esa mayor que $99 \%$ de confianza (valor- $p<0.01$ ). Aunque los valores de $\mathrm{SE}$ sean bajos, el valor de la temperatura de $30{ }^{\circ} \mathrm{C}$ del modelo de Oswin está arriba de lo esperado (>14\%), tornándolo poco satisfactorio para estimar esa isoterma en las condiciones estudiadas, pues ese debe ser inferior a $10 \%$ como establecido por Lomauro et al.
(1985). Entretanto, todo indica que los modelos de Smith y Peleg (1993) se ajustaron adecuadamente a las isotermas por mostrar valores de entre 0.4 y $7 \%$ y valores de SE entre 0 y 1.1. El modelo de Peleg (1993) presentó valores de SE por lo menos dos y tres veces inferiores a los encontrados para los modelos de Oswin y Smith, respectivamente, significando que se trata del modelo más adecuado para estimar la humedad de equilibrio de la harina mixta extrudida de arroz y bagazo de cebada en diferentes actividades de agua y temperaturas estudiadas.

Como pode ser visto, los dos modelos matemáticos de Peleg fueron seleccionados para estimar la humedad de equilibrio de adsorción de agua de la muestra estudiada. Ambos modelos presentaron una buena estadística de validación, sin embargo, el modelo de Peleg (1988) sobrestima los valores de $\mathrm{X}_{\mathrm{e}}$, en cuanto que el modelo de Peleg (1993) presenta los menores residuos cuando comparado con los datos observados (Figura 3). En los gráficos de residuos se pode observar que el modelo de Peleg (1988) linealizado presenta residuos positivos y sus valores tienden a aumentar con el incremento de $\mathrm{X}_{\mathrm{e}}$, contrariamente, los residuos presentados por el modelo de Peleg (1993) se distribuyeron en torno de la media de los residuos. Con estas observaciones concluyese que el modelo matemático de Peleg (1993) es el más adecuado para predecir la humedad de equilibrio de la harina mixta extrudida de arroz y bagazo de cebada.

La lectura completa del RVA de las pastas de harina de arroz y mixta extrudida de arroz y bagazo de cebada está diseñada en la Figura 4. En el perfil de viscosidad de estas harinas se observan las modificaciones de los gránulos de almidón debido a la temperatura y al tiempo de agitación en el RVA.

La formación de la pasta del arroz se inicia a los 6 min de cocción a $79.9{ }^{\circ} \mathrm{C}$. Aquí, los gránulos de almidón comienzan a hinchar, aumentando la viscosidad hasta alcanzar un máximo de $1294 \mathrm{cP}$, registrada a los $8 \mathrm{~min}$ a una temperatura de $95{ }^{\circ} \mathrm{C}$. La quiebra de la viscosidad se detectó aún en el ciclo de calentamiento, después de 10 min de cocción, disminuyendo $196 \mathrm{cP}$ el valor de la viscosidad máxima, debido al rompimiento de los gránulos de almidón de arroz. La retrogradación de la pasta de arroz se inició aún en el ciclo de resfriamiento $90{ }^{\circ} \mathrm{C}$, donde la viscosidad fue aumentando a medida que la pasta se fue enfriando hasta el final del experimento, alcanzando la viscosidad final de $3863 \mathrm{cP}$, registrándose la viscosidad de retrogradación de $2765 \mathrm{cP}$.

Un perfil de viscosidad semejante a este fue descrito por Zhou et al. (2002) en el estudio de la composición y de las propiedades funcionales del arroz; por Sacchetti et al. (2004) para harina de arroz cruda, por Ascheri et al. (2006a) para harina de arroz y por Silva \& Ascheri (2009) para afrecho de arroz. 

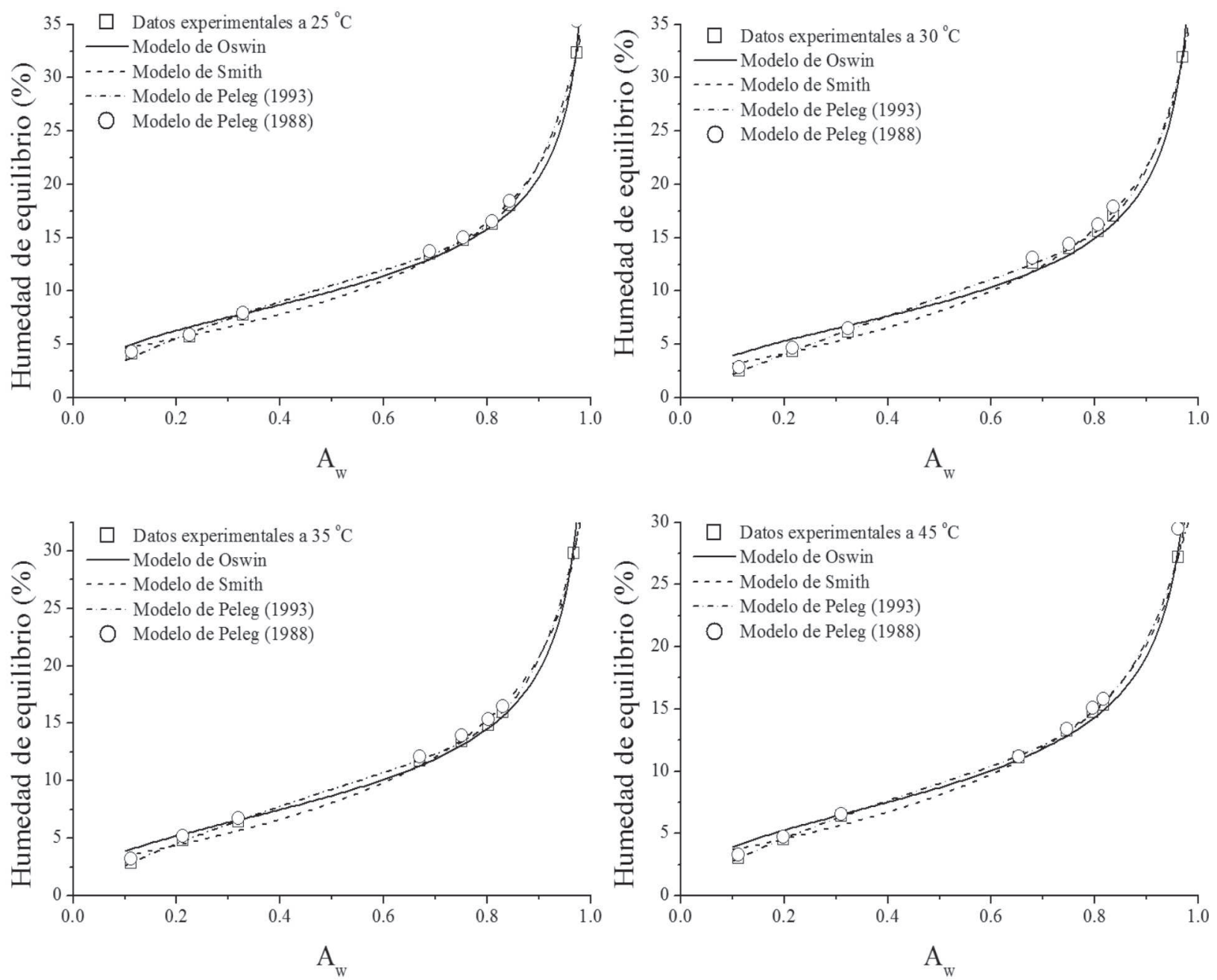

Figura 2. Curvas de humedad de equilibrio de la harina mixta extrudida de arroz y bagazo de cebada en función de la actividad de agua $\left(\mathrm{A}_{\mathrm{w}}\right)$, obtenidas en diferentes temperaturas.

Tabla 3. Constantes estimados de los modelos matemáticos de adsorción de agua y estadísticas de adecuación para estimar la humedad de equilibrio de la harina mixta extrudida de arroz y bagazo de cebada.

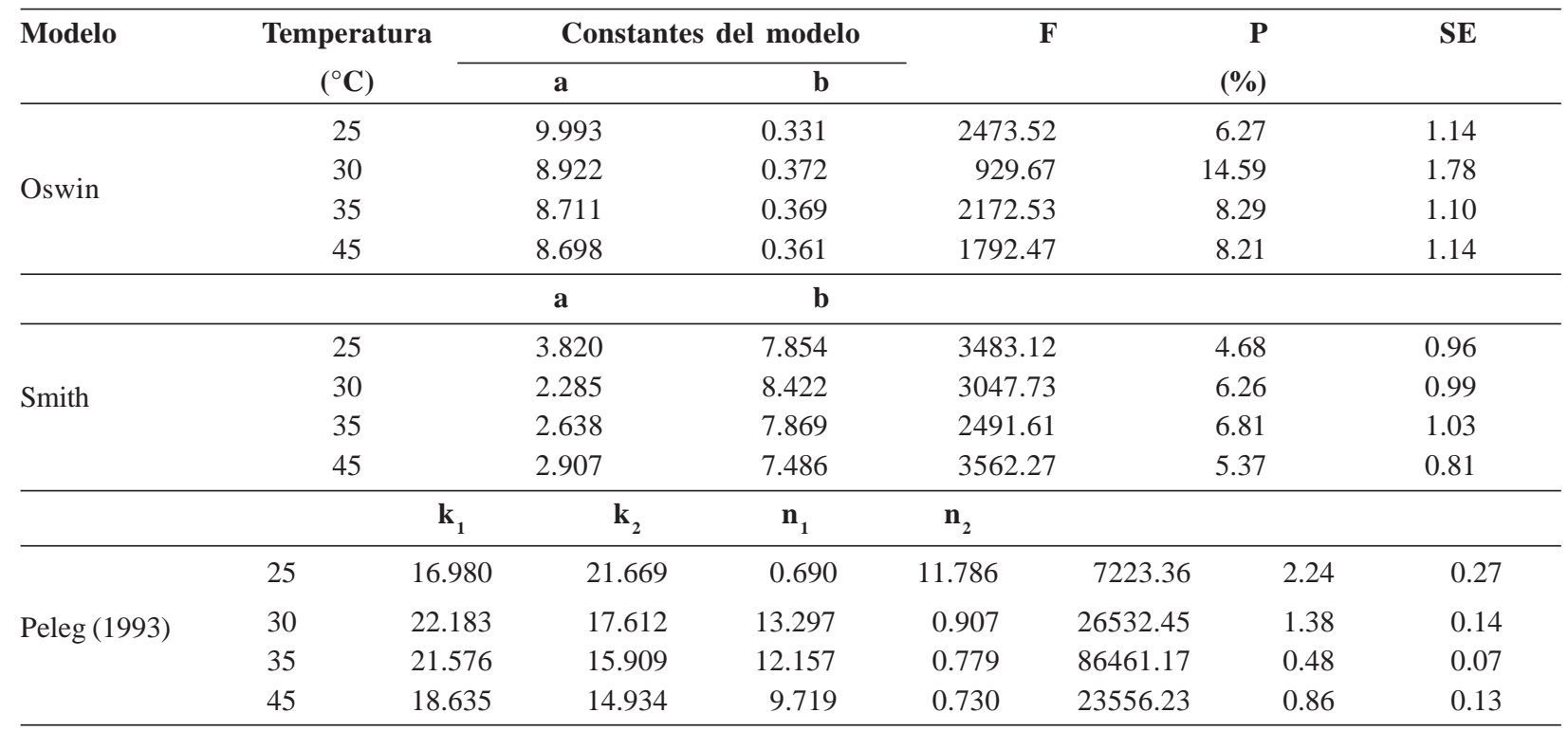

$\mathrm{a}, \mathrm{b}, \mathrm{k}_{1}, \mathrm{k}_{2}, \mathrm{n}_{1}$ y $\mathrm{n}_{2}$ : constantes características de las ecuaciones de adsorción de agua, $\mathrm{F}$ : valor del teste $\mathrm{F}, \mathrm{P}(\%)$ : error relativo medio y SE: error estándar del estimado. 
La harina mixta extrudida de arroz y bagazo de cebada presentó similar perfil de viscosidad que presentó el arroz crudo (Figura 4), con menores valores de temperatura de pasta $\left(59.5^{\circ} \mathrm{C}\right)$, quiebra de viscosidad $(79 \mathrm{cP})$ y viscosidad de retrogradación $(1163 \mathrm{cP})$, sin la presencia de la viscosidad a frío (a $25^{\circ} \mathrm{C}$ ). Este fenómeno indica que, aunque la muestra mixta tenga sufrido un drástico calentamiento durante la extrusión, el proceso solamente gelatinizó parcialmente el almidón contenido en el arroz, disminuyendo la temperatura de pasta de la harina mixta extrudida, también observado por Gupta et al. (2008).
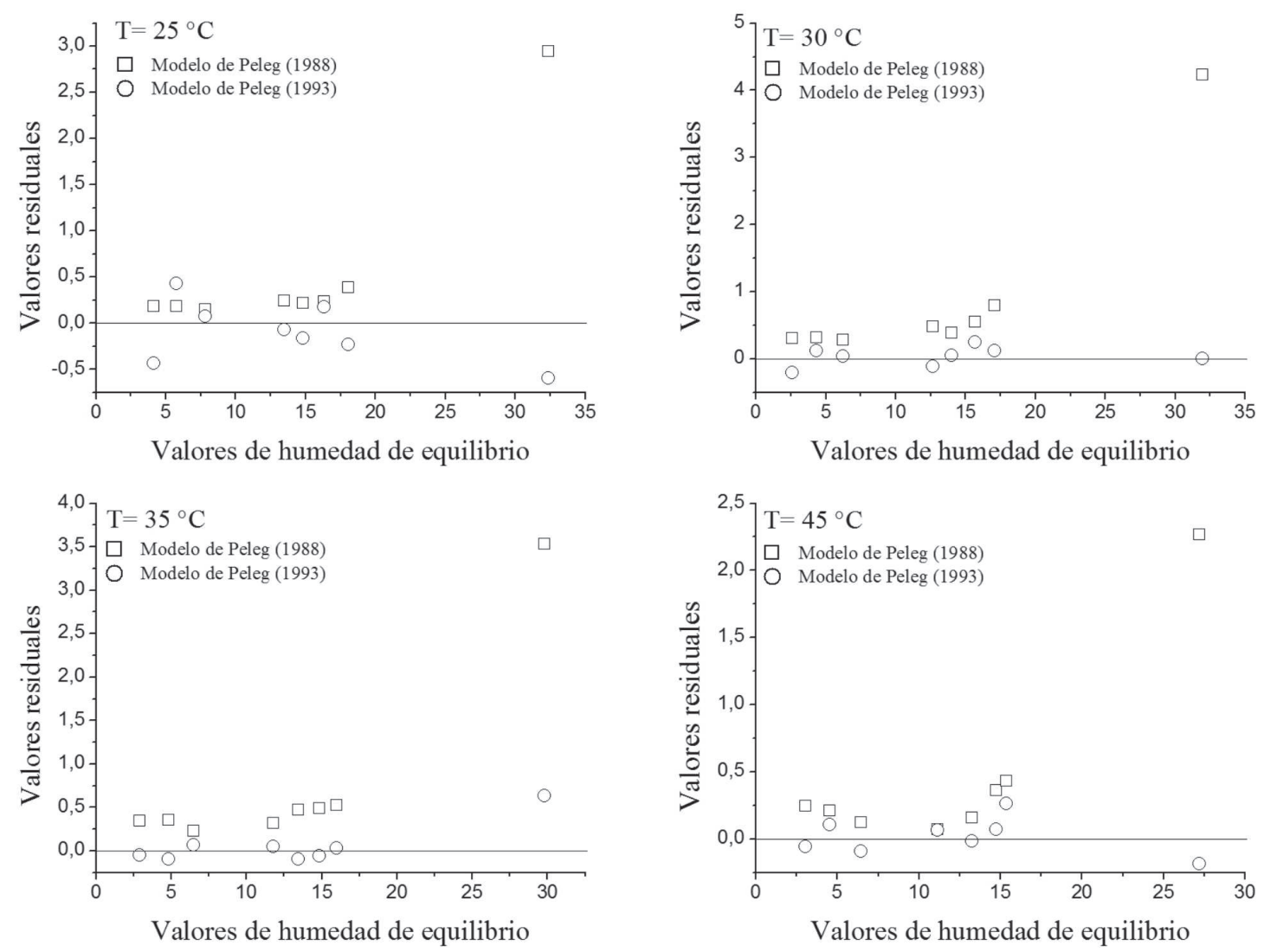

Figura 3. Valores de residuo medidos entre los valores experimentales de humedad de equilibrio de la harina mixta extrudida de arroz y bagazo de cebada y los valores estimados con los modelos matemáticos de Peleg (1988) y Peleg (1993), en diferentes temperaturas.

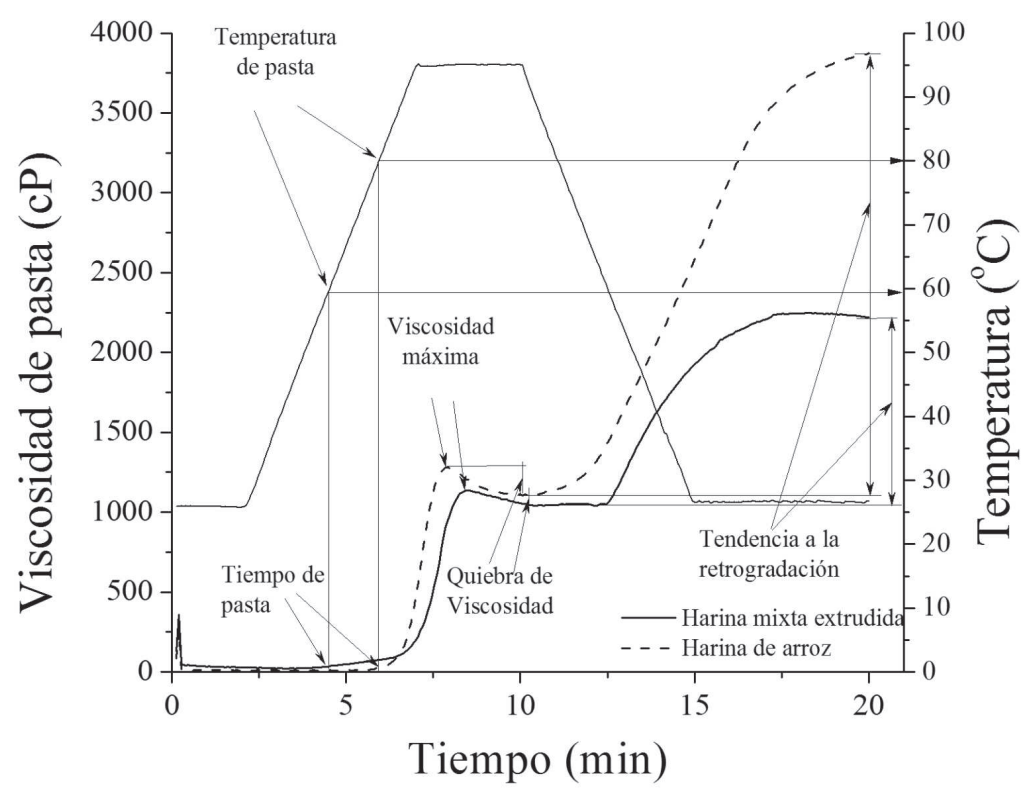

Figura 4. Perfil de viscosidad de la harina de arroz y de la harina mixta extrudida de arroz y bagazo de cebada. 
Caso contrario, la muestra extrudida podría comportarse como las de los estudios de Ascheri et al. (2006a), la viscosidad inicial (a $25^{\circ} \mathrm{C}$ ) de harinas mixtas extrudidas de arroz con bagazo de jabuticaba fue fuertemente influenciada por la formulación y por la velocidad de rotación del tornillo sin fin, seguidas por la temperatura, alcanzando viscosidades iniciales entre 200 a $700 \mathrm{cP}$, lo que no ocurrió en este estudio debido al bajo cizallamiento y alta humedad del procesamiento.

\section{CONCLUSIÓN}

En las condiciones operacionales del proceso de extrusión utilizadas en el presente trabajo, las fibras del bagazo de cebada reducen la gelatinización total del almidón contenido en el arroz, demostrado por la similitud de la curva de viscosidad de la pasta de la harina mixta extrudida con la harina de arroz crudo. La gelatinización parcial de la harina mixta extrudida se caracterizó por la baja temperatura de pasta, quiebra de viscosidad y tendencia a la retrogradación, los tres parámetros fueron menores que de la harina de arroz crudo.

El modelo de Peleg (1993) presentó mejor resultado en la predicción de las isotermas de adsorción de agua de la harina mixta de arroz y bagazo de cebada en las temperaturas de 25 a $45^{\circ} \mathrm{C}$ y actividades de agua entre 0.11 y 0.97 .

\section{AGRADECIMIENTOS}

Los autores agradecen a la Universidade Estadual de Goiás y a la Embrapa Agroindústria de Alimentos por el soporte técnico y a la Coordenação de Aperfeiçoamento de Pessoal de Nível Superior (Capes) por la bolsa de estudios concedida a la primera autora.

\section{REFERENCIAS}

Ascheri DPR, Pereira LD, Bassinello PZ, Carvalho CWP \& Ascheri JLR (2010) Calidad del arroz de tierras altas en función del tiempo de cocción y del cultivar de arroz. Scientia Agraria, 11:163-173.

Ascheri DPR, Andrade CT, Carvalho CWP \& Ascheri JLR (2006a) Obtenção de farinhas mistas pré-gelatinizadas a partir de arroz e bagaço de jabuticaba: efeito das variáveis de extrusão nas propriedades de pasta. Boletim do Centro de Pesquisa de Processamento de Alimentos, 24:115-144.

Ascheri DPR, Ascheri JLR \& Carvalho CWP (2006b) Caracterização da farinha de bagaço de jabuticaba e propriedades funcionais dos extrusados. Ciência e Tecnologia de Alimentos, 26:897905 .

Ascheri DPR, Moura WS, Ascheri JLR \& Freitas JEA (2009) Propriedades termodinâmicas de adsorção de água do amido de rizomas do lírio-do-brejo (Hedychium coronarium). Ciência e Tecnologia de Alimentos, 29:454-462.

Ascheri DPR, Nascimento GC \& Ascheri JLR (2003) Características de adsorción de agua de la harina de arroz soluble a varias temperaturas. Alimentaria, 40:111-119.
Ascheri DPR, Ribeiro M.M., Ascheri JLR \& Carvalho CWP (2005) Isotermas de adsorción de agua y estabilidad de harinas extrudidas de amaranto, arroz y maíz: estudio comparativo. Alimentaria, 363:100-107.

Baucour P \& Daudin JD (2000) Development of a new method for fast measurement of water sorption isotherms in the high humidity range: validation on gelatine gel. Journal of Food Engineering, 44:97-107.

Berglund PT, Fastnaught CE \& Holm ET (1994) Physicochemical and sensory evaluation of extruded high-fiber barley cereals. Cereal Chemistry, 71:91-95.

Chirife J \& Iglesias HA (1978) Equations for fitting water sorption isotherms of foods: Part 1 a review. Journal of Food Technology, 13:159-174.

Cunningham SE, Mcminn WAM \& Richardson PS (2007) Modelling water absoption of pasta during soaking. Journal of food Engineering, 82:600-607.

Elian SN (1988) Análise de Regressão. São Paulo, Instituto de Matemática e Estatística. 232 p.

García FP \& Martínez AJG (2006) Evaluación de las isotermas de sorción en cereales para desayuno. Superficies y Vacío, 19:1219.

Gupta M, Bawa AS \& Semwal AD (2008) Effect of barley flour on development of rice-based extruded snacks. Cereal Chemistry, $85: 115-122$.

Guy R (2001) Raw materials for extrusion cooking. In: Guy R (ed.) Extrusion cooking. Boca Raton, Woodhead Publishing. p. $5-28$.

International Union of Pure and Applied of Chemistry (1985) Reporting physisorption data for gas/solid systems. Pure and Applied Chemistry, 57:603-619.

Kulchan R, Boonsupthip W \& Suppakul P (2010) Shelf life prediction of packaged cassava-flour-based baked product by using empirical models and activation energy for water vapor permeability of polyolefin films. Journal of Food Engineering, 100, 461-467.

Labuza TP, Kaanane A \& Chen JY (1985) Effect of temperature on the moisture sorption isotherm and water activity shift of two dehydrated food. Journal Food Science, 50:392-396.

Lima CHAM, Ascheri DPR \& Bastos SMC (2012) Características de adsorção de água de farinhas cruas e pré-gelatinizadas de duas variedades de arroz de terras altas. Boletim do Centro de Pesquisa de Processamento de Alimentos, 30:91-102.

Lomauro CJ, Bakshi AS \& Labuza TP (1985) Evaluation of food moisture sorption isotherm equations. Parte 1: fruit vegetable and meat products. Lebensmittel Wissenschaft und Technology, 18:111-117.

Maia LH, Cornejo FEP, Ascheri DPR, Maia MCA \& Rocha-Leão MHM (2004) Moisture sorption studies on lactoferrin microcapsules. Alimentaria, 356: 79-87.

Montgomery DC \& Runger GC (2003) Estatística Aplicada e Probabilidade para Engenheiros. $2^{\mathrm{a}}$ ed. Rio de Janeiro, LTC. 514 p.

Peleg M (1988) An empirical model for the description of moisture sorption curves. Journal of Food Science, 53:1216-1217/1219.

Peleg M (1993) Assessment of a semi-empirical four parameter general model for sigmoid moisture sorption isotherms. Journal of Food Processing Engineering, 16:21-37.

Sacchetti G, Pinnavaia GG, Guidolin E \& Rosa MD (2004) Effects of extrusion temperature and feed composition on the functional, physical and sensory properties of chestnut and rice flour-based snack-like products. Food Research International, 37:527-534. 
Shuey WC \& Tipples KH (1980) The amylograph handbook. Saint Paul, AACC. 37 p.

Silva RF \& Ascheri JLR (2009) Extrusão de quirera de arroz para uso como ingrediente alimentar. Brazilian Journal of Food Technology, 12:190-199.

Turhan M, Sayar S \& Gunasekaran S (2002) Application of Peleg model to study water absorption in chickpea during soaking. Journal of Food Engineering, 53:153-159.

Van den Einde RM, Van der Veen ME, Bosman H, Van der Goot AJ \& Boon RM (2005) Modeling macromolecular degradation of corn starch in a twin screw extruder. Journal of Food Engineering, $66: 147-154$.
Yu Y \& Wang J (2007) Effect of ã-ray irradiation on starch granule structure and physicochemical properties of rice. Food Research International, 40:297-303.

Zanão CFP, Canniatti-Brazaca SG, Sarmento, SBS \& Arthur V (2009) Efeito da irradiação gama nas características físico-químicas e sensoriais do arroz (Oryza sativa L.) e no desenvolvimento de Sitophilus oryzae L. Ciência e Tecnologia de Alimentos, 29:46-55 